\title{
Mechanical Thrombectomy for Postoperative Stroke in a Patient with Acute Aortic Dissection Type A
}

\author{
Hiromu Kehara, ${ }_{1}^{1}$ Syuichi Urashita, ${ }^{2}$ Toshihito Gomibuchi, ${ }_{1}$ Kazunori Komatsu, ${ }^{1}$ Kouhei Takahashi, ${ }^{3}$ \\ Katsuaki Tsukioka, ${ }^{3}$ Takamitsu Terasaki, ${ }^{1}$ Tetsuya Kono, ${ }^{1}$ Naomichi Wada ${ }^{4}$ Yukinari Kakizawa, ${ }^{4}$ \\ Jun-ichi Koyama, ${ }^{5}$ and Kenji Okada ${ }^{6}$
}

\begin{abstract}
Mechanical thrombectomy has been proposed to expand the treatment time window and enhance revascularization. However, it is unclear whether its use can be extended to patients with occlusions in acute aortic dissection, especially the thoracic aorta. A 55-year-old man underwent graft replacement for acute aortic dissection type A. On postoperative day 2 , he developed stroke and computed tomography showed occlusion of the right middle cerebral artery. Mechanical thrombectomy was performed by transbrachial approach. Although successful recanalization was achieved, he suffered hemorrhagic stroke. Since there is no other effective treatment and the neurologic outcome with conservative management is poor, we consider mechanical thrombectomy to be a viable therapeutic option for the treatment of postoperative stroke in patients with acute aortic dissection type A. However, further study is warranted regarding the safety of this technique.
\end{abstract}

Keywords: mechanical thrombectomy, acute aortic dissection type A, transbrachial approach

\section{Introduction}

Nowadays mechanical thrombectomy, which is endovascular therapy for arterial occlusion in cardiogenic stroke, has been proposed to expand the treatment time window and enhance revascularization compared with thrombolysis alone, ${ }^{1)}$ and this new technique is expected to be used for early postoperative major stroke. However, it is unclear whether its use can be extended to patients with occlusions in acute

\footnotetext{
${ }^{1}$ Department of Cardiovascular Surgery, Suwa Red Cross Hospital, Suwa, Nagano, Japan

${ }^{2}$ Department of Cardiovascular Surgery, Kumamoto Red Cross Hospital, Kumamoto, Kumamoto, Japan

${ }^{3}$ Department of Cardiovascular Surgery, lida Municipal Hospital, lida, Nagano, Japan

${ }^{4}$ Department of Neurosurgery, Suwa Red Cross Hospital, Suwa, Nagano, Japan

${ }^{5}$ Neuroendovascular Therapy Center, Shinshu University Hospital, Matsumoto, Nagano, Japan

${ }^{6}$ Department of Cardiovascular Surgery, Kobe University, Kobe, Hyogo, Japan
}

Received: June 11, 2019; Accepted: October 18, 2019

Online March 24, 2020

Copyright $₫ 2020$ by The Japan Neurosurgical Society

This work is licensed under a Creative Commons AttributionNonCommercial-NoDerivatives International License. aortic dissection, especially the thoracic aorta. It is known that cerebral embolism occur due to aortic dissection other than cardiogenesis. The thrombolysis for cerebral embolism by aortic dissection is contraindicate, and thrombectomy using transfemoral approach is usually not performed because of residual dissection.

Although there is a report of mechanical embolectomy in the setting of aortic dissection, the cases involved two chronic aortic dissection type $\mathrm{A}$ and one acute aortic dissection type B. $^{2)}$ To the best of our knowledge, there have been no previously reported cases of mechanical embolectomy in patients with acute aortic dissection type A (AADA).

We herein report a case of mechanical embolectomy in a patient with postoperative stroke after graft replacement for AADA.

\section{Case Report}

A 55-year-old man was diagnosed with AADA and transferred to our hospital. Computed tomography (CT) revealed a tear at the proximal arch and severe stenosis of the right common carotid artery (CCA) excluded by a thrombosed false lumen (Fig. 1A). Neurologic examination was negative. The patient underwent graft replacement with a Dacron graft. Cardiopulmonary bypass was performed via the right axillary artery and left femoral artery, and cannulation of the right atrium. A distal anastomosis was constructed between the brachiocephalic artery (BCA) and left CCA under the conditions of moderately hypothermic circulatory arrest with selective cerebral perfusion. The BCA was also dissected, and fixed with glue and anastomosed to a branch of the prosthetic graft. On postoperative day (POD) 1, the patient was weaned from the respirator with no neurologic symptoms. On POD 2, however, right conjugate deviation and left hemiplegia were observed, with modified Rankin scale (mRS) 5. CT showed obstruction of the right CCA (Fig. 1B) and thrombus at the right middle cerebral artery (MCA) (Fig. 1C). Given no obvious infarct on head CT, right MCA occlusion, and persistent severe neurologic deficit, our multidisciplinary team determined that the possible benefits of endovascular intervention outweighed the risks, and the patient was taken emergently for mechanical thrombectomy. Given that there was residual acute aortic dissection over the distal anastomotic site, the transfemoral approach was thought to be dangerous. A right transbrachial approach was used percutaneously with a 9-Fr sheath, with subsequent uncomplicated access to 


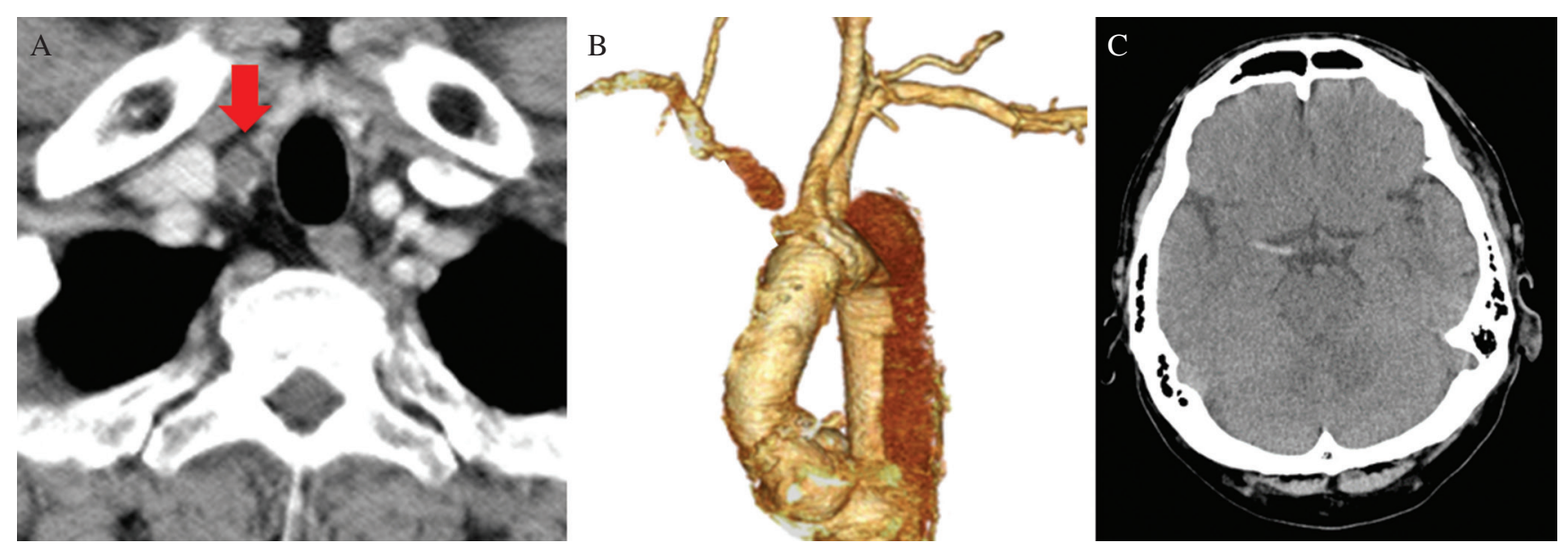

Fig. 1 Preoperative computed tomography (CT) scan shows severe stenosis of right common carotid artery excluded by a thrombosed false lumen (red arrow, A). Postoperative 3D CT shows that the side branch of the prosthetic graft, the BCA, and the right CCA were not enhanced (B), and head $\mathrm{CT}$ scan shows occlusion of the right MCA (C). BCA: brachiocephalic artery, CCA: common carotid artery, MCA: middle cerebral artery.
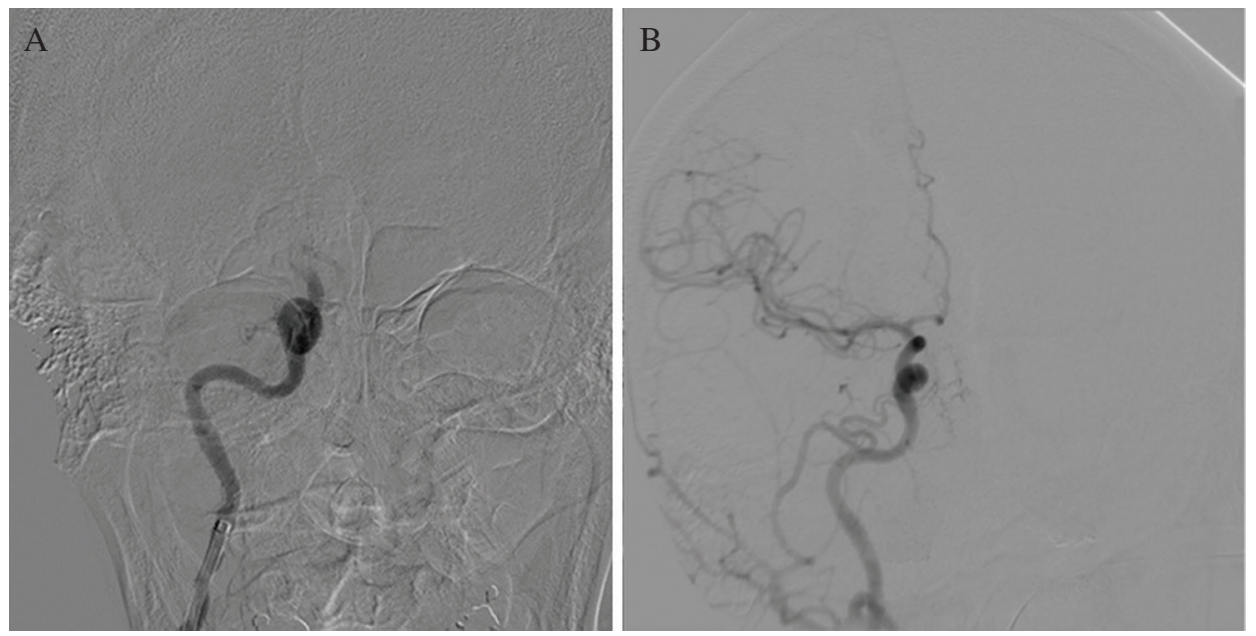

Fig. 2 Angiography showed obstruction of right internal carotid artery (A). After the mechanical thrombectomy, successful recanalization was achieved, consistent with thrombolysis in cerebral infarction (TICI) 3 flow (B).

the right MCA occlusion using 9-Fr OPTIMO balloon guiding catheter (Tokai Medical Products Inc., Kasugai, Aichi, Japan), a Marksman microcatheter (Medtronic, Minneapolis, MN, USA) and a 0.014-inch Chikai microwire (Asahi Intecc Co., Ltd., Seto, Aichi, Japan). Solitaire FR $6 \times 30$-mm (Covidien, Irvine, CA, USA) was then deployed and retrieved with the clot under manual aspiration of the balloon guiding catheter. Successful recanalization was achieved, consistent with thrombolysis in cerebral infarction (TICI) three flow after $4.5 \mathrm{~h}$ from onset (Fig. 2), but the right CCA flow was poor because of false lumen expansion (Fig. 3), so we decided to add stent to prevent recurrence. Under general anesthesia, we exposed the right CCA. After the right CCA was clamped, arteriotomy was done and a 6-Fr sheath was inserted through the true lumen under direct vision. A 0.035 -inch guide wire was passed into the graft in retrograde fashion, and a $12 \times 60-\mathrm{mm}$ Epic Vascular SelfExpanding Stent (Boston Scientific, Natick, MA, USA) was deployed. Repeat angiography (Fig. 3) and 3D CT (Fig. 1D) showed restitution of the right CCA blood flow. The endovascular details are summarized in Fig. 4. Unfortunately, postoperative cerebral CT documented right-sided hemorrhagic stroke (Fig. 5). On POD 74, the patient was discharged to another hospital for further rehabilitation with mRS 4 . Twenty months after the operation he can walk by himself without a cane, the mRS had improved to 2 , and no recurrence of stroke has been observed.

\section{Discussion}

Our case suggests that we could recanalize the MCA using a transbrachial approach in AADA patients with subsequent uncomplicated access. Catheterization in patients with aortic dissection has been considered technically difficult, time consuming, and potentially risky. Moreover, there is also the risk that advancing the catheter or guide wire may cause extension of the dissection, displacement of thrombotic material, or outright perforation of the aorta. ${ }^{3)}$ The access site is a major consideration and must be decided on a case-by-case basis. The major factors influencing choice of access site include the location of the dissection as well as the location of the target vessel. There are three access routes: transfemoral, transcarotid, and transbrachial. An acute 

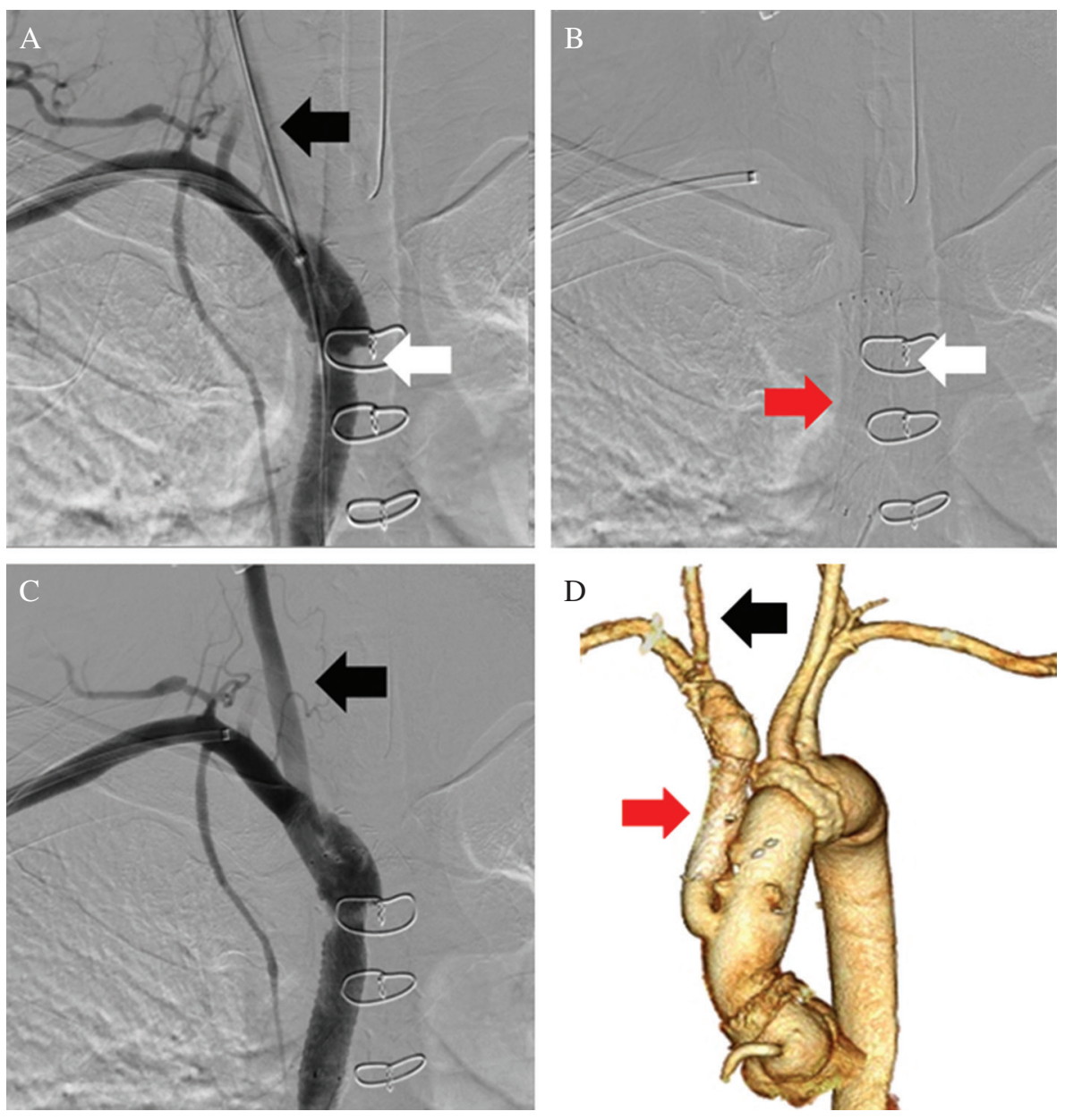

Fig. 3 Angiography showed severe stenosis at anastomotic site between branch of the graft and the brachiocephalic artery (white arrow) and the right common carotid artery (CCA) flow was poor (black arrow, A). Stent (red arrow) was deployed across the anastomotic site (white arrow, B). Repeat angiography showed resolution of the right CCA blood flow (black arrow, C). After stenting (red arrow), 3D computed tomography shows restitution of the right CCA blood flow (black arrow, D).

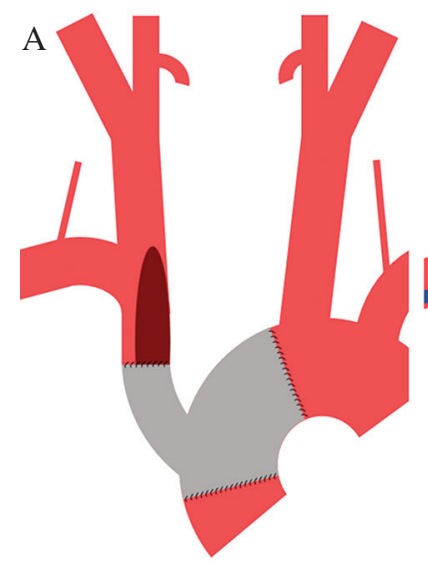

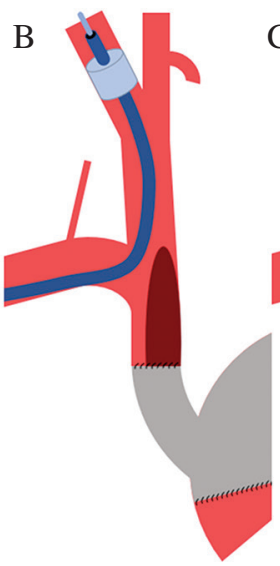

C

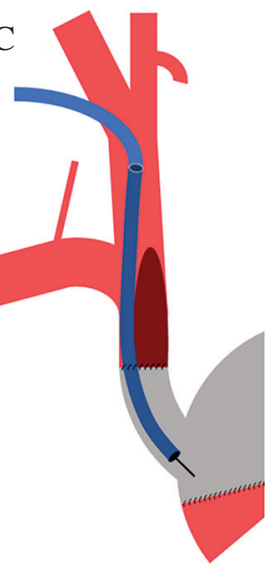

$\mathrm{D}$

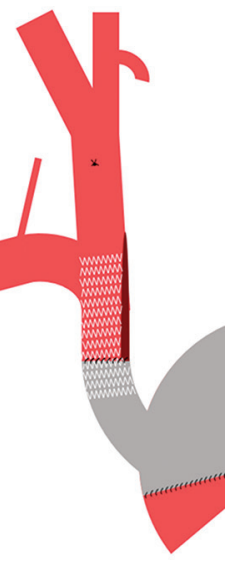

Fig. 4 Illustrations of mechanical thrombectomy and stenting. Brown part is thrombosed false lumen and gray part is prosthetic graft. Postoperatively, BCA and right common carotid artery (CCA) was excluded by thrombosed false lumen (A). Mechanical thrombectomy was performed using transbrachial approach through true lumen at bifurcation of right CCA and right subclavian artery (B). CCA was exposed, sheath was inserted through the true lumen under direct vision into graft (C). Stent was deployed across anastomotic site (D). BCA: brachiocephalic artery. aortic dissection may prohibit a transfemoral approach, whereas a more chronic dissection would likely be healed, making a transfemoral approach more technically feasible. Meanwhile, if the target vessel is in the anterior circulation of the brain, a direct carotid approach may be favorable, although closure is a challenge especially in uncooperative patients. ${ }^{4)}$ When there is concern about hemostasis, cutdown and exposure of the carotid artery is very useful although general anesthesia might be needed. Even though the size of catheter is limited, a transbrachial approach may be more appropriate because involvement of an axillary artery by the dissection process is rare. ${ }^{5)}$

The major adverse effect of this technique is hemorrhagic transformation, and the occurrence is mostly time dependent. ${ }^{6}$ In the present case it took only $4.5 \mathrm{~h}$ to remove the clot. Although hemorrhagic stroke occurred in this patient, since there was no other effective treatment and the neurologic outcome with conservative management would be very poor, we considered mechanical embolectomy to be a viable therapeutic option in treating postoperative massive stroke in 


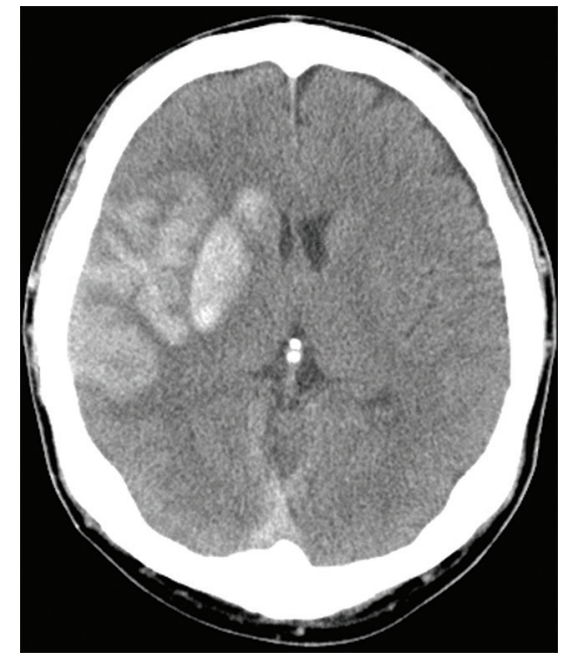

Fig. 5 Cerebral CT after mechanical thrombectomy and stenting showed right-sided hemorrhagic stroke. CT: computed tomography.

this AADA patient. It is unknown whether hemorrhagic transformation is related to aortic dissection, and further study is warranted regarding the time limit and safety of this technique.

Persistent CCA dissection (CCAD) from extension of AADA after graft replacement is not rare, the prevalence being reported as $15-24 \%$. Most studies with large series reported good neurologic outcomes without the need for CCAD intervention. ${ }^{78}$ Zieliński et al. ${ }^{7)}$ reported that the majority of cases showed no progression and patients remained asymptomatic during a 2-year follow-up by carotid ultrasound. Charlton-Ouw et al. ${ }^{8}$ recently reported a series of 179 AADA patients in which the postoperative stroke rate was not significantly different between CCAD (14.6\%) and without CCAD (10.3\%). However, there is a subgroup of patients who develop severe stroke symptoms as a result of carotid artery occlusion, eventually in combination with thromboembolic obstruction of the central arteries. ${ }^{9)}$ The pathogenesis of stroke in this case was unclear, but we speculate it was thromboembolism from false lumen as described above. We thought prophylactic treatment for CCAD after graft replacement of AADA is controversial. When a stroke occurs, prompt mechanical thrombectomy and/or stenting are warranted depending on the situation.

\section{Acknowledgments}

We thank Hugh McGonigle, from Edanz Group (www. edanzediting.com/ac), for editing a draft of the manuscript.

\section{Conflicts of Interest Disclosure}

All authors have no conflicts of interest.

\section{References}

1) Dávalos A, Pereira VM, Chapot R, et al.: Retrospective multicenter study of Solitaire FR for revascularization in the treatment of acute ischemic stroke. Stroke 43: 2699-2705, 2012

2) Reznik ME, Espinosa-Morales AD, Jumaa MA, Zaidi S, Ducruet AF, Jadhav AP: Endovascular thrombectomy in the setting of aortic dissection. J Neurointerv Surg 9: 17-20, 2017

3) Hart WL, Berman EJ, LaCom RJ: Hazard of retrograde aortography in dissecting aneurysm. Circulation 27: 1140-1142, 1963

4) Jadhav AP, Ribo M, Grandhi R, et al.: Transcervical access in acute ischemic stroke. J Neurointerv Surg 6: 652-657, 2014

5) Borst HG: In Borst HG, Heinemann MK, Stone CD (eds): Surgical treatment of aortic dissection, ed 1. New York, Churchill Livingstone, 1996, pp. 255-268

6) Kaesmacher J, Kaesmacher M, Maegerlein C, et al.: Hemorrhagic transformations after thrombectomy: risk factors and clinical relevance. Cerebrovasc Dis 43: 294-304, 2017

7) Zieliński T, Wołkanin-Bartnik J, Janaszek-Sitkowska H, et al.: Persistent dissection of carotid artery in patients operated on for type A acute aortic dissection-carotid ultrasound follow-up. Int J Cardiol 70: 133-139, 1999

8) Charlton-Ouw KM, Azizzadeh A, Sandhu HK, et al.: Management of common carotid artery dissection due to extension from acute type A (DeBakey I) aortic dissection. J Vasc Surg 58: 910-916, 2013

9) Kwak HS, Hwang SB, Jin GY, Hippe DS, Chung GH: Predictors of functional outcome after emergency carotid artery stenting and intraarterial thrombolysis for treatment of acute stroke associated with obstruction of the proximal internal carotid artery and tandem downstream occlusion. AJNR Am J Neuroradiol 4: 841-846, 2013 